\title{
Large quantum superpositions of a levitated nanodiamond through spin-optomechanical coupling
}

\author{
Zhang-qi Yin, ${ }^{1, \text { * }}$ Tongcang Li, ${ }^{2}$ Xiang Zhang, ${ }^{2}$ and L. M. Duan ${ }^{3,4}$ \\ ${ }^{1}$ The Center for Quantum Information, Institute for Interdisciplinary Information Sciences, \\ Tsinghua University, Beijing 100084, P. R. China \\ ${ }^{2}$ NSF Nanoscale Science and Engineering Center, 3112 Etcheverry Hall, \\ University of California, Berkeley, California 94720, USA \\ ${ }^{3}$ The Center for Quantum Information, Institute for Interdisciplinary Information Sciences, \\ Tsinghua University, Beijing 100084, People's Republic of China \\ ${ }^{4}$ Department of Physics, University of Michigan, Ann Arbor, Michigan 48109, USA
}

(Dated: September 13, 2013)

\begin{abstract}
We propose a method to generate and detect large quantum superposition states and arbitrary Fock states for the oscillational mode of an optically levitated nanocrystal diamond. The nonlinear interaction required for the generation of non-Gaussian quantum states is enabled through the spinmechanical coupling with a built-in nitrogen-vacancy center inside the nanodiamond. The proposed method allows the generation of large superpositions of nanoparticles with millions of atoms and the observation of the associated spatial quantum interference under reasonable experimental conditions.
\end{abstract}

\section{INTRODUCTION}

Creating spatial quantum superpositions with massive objects is one of the most challenging and attractive goals in macroscopic quantum mechanics 1 - 4$]$. It provides potential opportunities to experimentally test different wave-function collapse models 2], including gravity-induced state reduction [5], which is a manifestation of the apparent conflict between general relativity and quantum mechanics. Quantum superpositions and interferences have been realized with electrons, neutrons, atoms, and complex molecules with several hundred atoms [6]. Among different optomechanical systems 7-10], optically levitated dielectric particles in vacuum [11 21] are particularly promising for creating superposition states with the largest macroscopicity (as defined in Ref. [4]). Due to the absence of the mechanical contact in this system, the decoherence 22] can be negligible and the oscillation frequency is fully tunable. Once cooled to the quantum regime, optically trapped nanoparticles in vacuum will be ultra-sensitive detectors [23 25], and can be even used to study self-assembly of the nanoparticles in vacuum [26, 27]. To generate spatial quantum superpositions and other non-Gaussian states with an optical cavity, however, requires a very strong quadratic coupling [21, 28]. This is a very demanding requirement. To enhance the quadratic coupling, Romero-Isart et al. 21. proposed to prepare spatial quantum superpositions of nanoparticles with two inter-connected high-finesse optical cavities: one cavity for cooling, and the other cavity for preparing the superposition state with a squared position measurement when the nanoparticle falls through it.

In this paper, we propose a scheme to generate and

*yinzhangqi@mail.tsinghua.edu.cn detect arbitrary Fock states and spatial quantum superposition states for the center-of-mass oscillation of an optically trapped nanocrystal diamond using the induced spin-opto-mechanical coupling. The nanodiamond has build-in nitrogen-vacancy (NV) centers, and electron spins associated with diamond NV centers make good qubits for quantum information processing as they have nice coherence properties even at room temperature 29]. With assistance of a strong magnetic field gradient from a nearby magnetic tip, strong coupling between the NV spin and the mechanical oscillation of the nanodiamond can be achieved. Using this coupling, we show how to generate arbitrary Fock states, entangled states, and large quantum superpositions for the nanodiamond. The generated spatial superposition states and other mesoscopic quantum superposition states can be detected through different spatial interference patterns of the nanodiamond.

\section{THE PROPOSED SETUP}

Nanodiamonds with NV centers have been recently trapped by optical tweezers in liquid [30, 31] and atmospheric air [32], and similar technologies can be used to optically trap them in vacuum [16]. As shown in Fig. 1, we consider a nanodiamond of mass $m$ optically trapped in vacuum with trapping frequency $\omega_{m}$. The motion of its center-of-mass mode $a_{m}$ is described by the Hamiltonian $H_{m}=\hbar \omega_{m} a_{m}^{\dagger} a_{m}$. The nanodiamond has a built-in NV center with its level configuration shown in Fig.1b in the ground-state manifold. The NV spin is described by the Hamiltonian $H_{\mathrm{NV}}=\hbar\left(\omega_{+1}|+1\rangle\left\langle+1\left|+\omega_{-1}\right|-1\right\rangle\langle-1|\right)$, where we have set $|0\rangle$ as the energy zero point. A magnet tip near the NV center induces a strong magnetic field gradient [33, 34], which couples the electron spin and the center-of-mass oscillation of the nanodiamond. The coupling Hamiltonian is denoted by $H_{\mathrm{NV} m}=\hbar \lambda S_{z}\left(a_{m}+a_{m}^{\dagger}\right)$ 
(a)

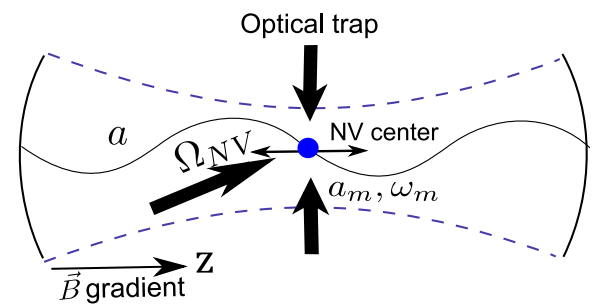

(b)
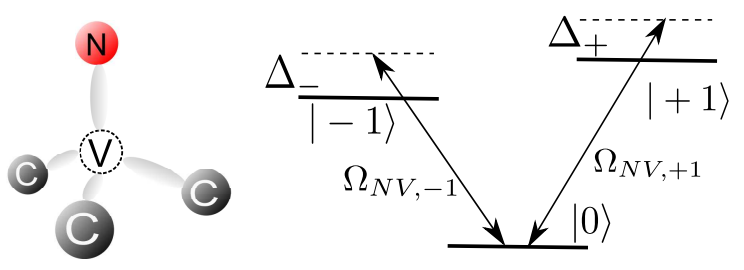

FIG. 1: (Color online) (a) A nanodiamond with a NV center is optically trapped in vacuum with spin-mechanical coupling enabled through a nearby magnetic tip and opto-mechanical coupling through a cavity around. (b) The atomic structure (left) and the level diagram (right) in the ground state manifold for a NV center in the nanodiamond .

[35, 36], where $S_{z} \equiv|+1\rangle\langle+1|-|-1\rangle\langle-1|$. The coupling strength $\lambda=g_{s} \mu_{B} G_{m} a_{0} / \hbar$, where $a_{0}=\sqrt{\hbar / 2 m \omega_{m}}$, $g_{s} \simeq 2$ is the Landé g-factor, $\mu_{B}$ is the Bohr magneton, and $G_{m}$ is the magnetic field gradient along the NV center axis.

The nanodiamond is trapped inside an optical cavity to pre-cool its center-of-mass motion to the ground state through the cavity-assisted cooling as has been demonstrated for other mechanical systems [9]. The heating of the mechanical mode is negligible compared with the cavity-induced cooling rate as the $Q$-factor for the centerof-mass oscillation of an optically levitated particle in vacuum is very high 11]. Note that the center-mass oscillation is not subject to the intrinsic dissipation of the material as it is decoupled from all the other mechanical modes and can be cooled to the ground state even if the diamond itself (its internal modes) is at room temperature. An alternative way for the ground state cooling of the center-of-mass mode, although not demonstrated yet in experiments, is to use a combination of optical pumping of the NV spin state and fast exchange between the spin and the motional excitations [35]. In this case, we do not need any optical cavity, which can further simplify the experimental setup.

\section{PREPARATION AND DETECTION OF FOCK STATES}

In order to prepare the Fock states, we first cool the mechanical mode to the ground state. The NV spin is initially set to the state $|0\rangle$, which is decoupled from the mechanical mode during the cooling. Initialization and single shot detection of the NV spin have been well accomplished experimentally [37]. We assume that the NV center is at a position with zero magnetic field and a large field gradient. We apply a microwave drive with the Hamiltonian $H_{\text {drive }}=\hbar\left(\Omega_{\mathrm{NV},+1} e^{i \omega_{l+} t}|0\rangle\langle+1|+\right.$ $\Omega_{\mathrm{NV},-1} e^{i \omega_{l}-t}|0\rangle\langle-1|+$ h.c. $) / 2$ and set the Rabi frequency $\Omega_{\mathrm{NV}, \pm 1}=\Omega_{\mathrm{NV}}$ and the detuning $\Delta_{ \pm} \equiv \omega_{l \pm}-\omega_{ \pm 1}=\Delta$. With $\Delta \gg\left|\Omega_{\mathrm{NV}}\right|$, we adiabatically eliminate the level $|0\rangle$ and get the following effective Hamiltonian

$$
H_{e}=\hbar \omega_{m} a_{m}^{\dagger} a_{m}+\hbar \Omega \sigma_{z}+\hbar \lambda\left(\sigma_{+}+\sigma_{-}\right)\left(a_{m}+a_{m}^{\dagger}\right)
$$

where $\Omega=\left|\Omega_{\mathrm{NV}}\right|^{2} / 4 \Delta, \sigma_{z}=|+\rangle\langle+|-|-\rangle\langle-|, \sigma_{+}=$ $|+\rangle\left\langle-\left|, \sigma_{-}=\right|-\right\rangle\langle+|$, and we have defined the new basis states $|+\rangle=(|+1\rangle+|-1\rangle) / \sqrt{2},|-\rangle=(|+1\rangle-\mid-$ $1\rangle) / \sqrt{2}$. In the limit $\lambda \ll \omega_{m}$, we set $\Omega=\omega_{m} / 2$ and use the rotating wave approximation to get an effective interaction Hamiltonian between the mechanical mode and the NV center spin, with the form

$$
H_{J C}=\hbar \lambda \sigma_{+} a_{m}+h . c . .
$$

This represents the standard Jaynes-Cummings(J-C) coupling Hamiltonian. Similarly, if we set $\Omega=-\omega_{m} / 2$, the anti J-C Hamiltonian can be realized with

$$
H_{a J C}=\hbar \lambda \sigma_{+} a_{m}^{\dagger}+h . c . .
$$
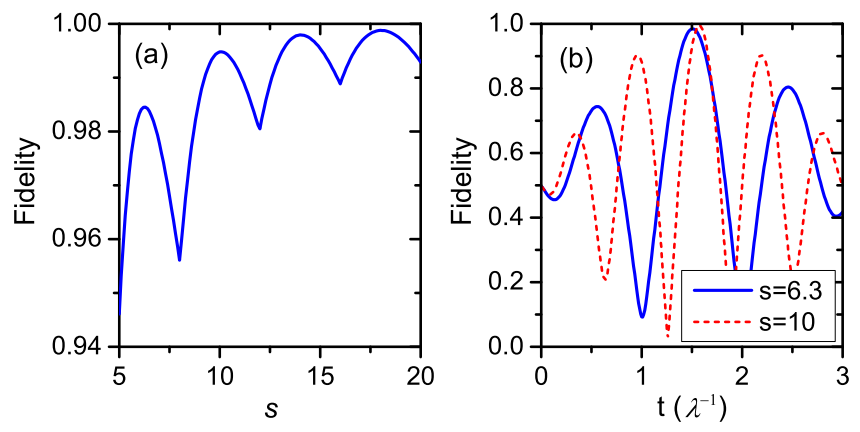

FIG. 2: (Color online) Fidelity of creating the phonon number superpostion state $\left(|0\rangle_{m}+i|1\rangle_{m}\right) / \sqrt{2}$ by coherent state transfer between the NV spin and the mechanical mode. Fidelities higher than $99 \%$ can be achieved. (a) The peak fidelity as a function of the parameter $s=\omega_{m} / \lambda$. (b) The fidelity as a function of the interaction time for two different parameters $(s=6.3,10)$.

Arbitrary Fock states and their superpositions can be prepared with a combination of $\mathrm{J}-\mathrm{C}$ and anti $\mathrm{J}-\mathrm{C}$ coupling Hamiltonians. For example, to generate the Fock state $|2\rangle_{m}$, we initialize the state to $|+\rangle|0\rangle_{m}$, turn on the J-C coupling for a duration $t_{1}=\pi /(2 \lambda)$ to get $|-\rangle|1\rangle_{m}$, and then turn on the anti J-C coupling for a duration $t_{2}=t_{1} / \sqrt{2}$ to get $|+\rangle|2\rangle_{m}$. The Fock state with arbitrary phonon number $n_{m}$ can be generated by repeating the above two basic steps, and the interaction time is $t_{i}=t_{1} / \sqrt{i}$ for the $i$ th step [38]. Superpositions of different Fock states can also be generated. For instance, if we initialize the state to $\left(c_{0}|+\rangle+c_{1}|-\rangle\right) \otimes|0\rangle_{m} / \sqrt{2}$ through a microwave with arbitrary coefficients $c_{0}, c_{1}$, and turn on 
the J-C coupling for a duration $t_{1}$, we get the superposition state $|-\rangle \otimes\left(c_{1}|0\rangle_{m}+i c_{0}|1\rangle_{m}\right) / \sqrt{2}$. In Fig. 2a, we plot the fidelity of the mechanical state as a function of the parameter $s=\omega_{m} / \lambda$ using the full Hamiltonian with rotating wave approximation. The fidelity oscillates with many local maxima and the envelope approaches unity when $s \gg 1$. In practice, we have a very high fidelity already by setting $s$ at the local maxima such as 6.3 or10.0. Using the optical cavity, the Fock state $\left|n_{m}\right\rangle_{m}$ of mechanical mode can also be mapped to the corresponding Fock state of the output light field [25].

The effective Hamiltonian for the spin-phonon coupling takes the form

$$
H_{Q N D}=\hbar \chi \sigma_{z} a_{m}^{\dagger} a_{m}
$$

with $\chi=4 \Omega \lambda^{2} /\left(4 \Omega^{2}-\omega_{m}^{2}\right)$ when the detuning ||$\Omega \mid-$ $\omega_{m} / 2 \mid \gg \lambda$. The Hamiltonian $H_{Q N D}$ can be used for a quantum non-demolition measurement(QND) measurement of the phonon number: we prepare the NV center spin in a superposition state $\left.|+\rangle+e^{i \phi}|-\rangle\right) / \sqrt{2}$, and the phase $\phi$ evolves by $\phi(t)=\phi_{0}+2 \chi n_{m} t$, where $n_{m}=a_{m}^{\dagger} a_{m}$ denotes the phonon number. Through a measurement of the phase change, one can detect the phonon number.

The preparation and detection of the Fock states can all be done within the spin coherence time. Let us estimate the typical parameters. A large magnetic field gradient can be generated by moving the nanodiamond close to a magnetic tip. A large field gradient up to $4 \times 10^{7} \mathrm{~T} / \mathrm{m}$ has been reported in 2006 near the write head of a magnetic disk drive 33. In magnetic resonance force microscopy systems, the gradient in the order of $10^{6} \mathrm{~T} / \mathrm{m}$ has been realized in 2007 [34]. Here we take the gradient $G=10^{5} \mathrm{~T} / \mathrm{m}$ and get the coupling $\lambda \simeq 2 \pi \times 52 \mathrm{kHz}$ for a nanodiamond with the diameter $d=30 \mathrm{~nm}$ in an optical trap with a trapping frequency $\omega_{m}=2 \pi \times 0.5 \mathrm{MHz}$. The Fock states and their superpositions can then be generated with a time scale $1 / \lambda$ about a few $\mu$ s, and the QND detection rate $2|\chi| \sim 2 \pi \times 25 \mathrm{kHz}$ with the detuning ||$\Omega\left|-\omega_{m} / 2\right| \sim 5 \lambda$. The NV electron spin dephasing time over $1.8 \mathrm{~ms}$ has been observed at room temperature [39], which is long compared with the Fock state preparation time $1 / \lambda$ and the detection time $1 /(2|\chi|)$. The threshold gradient is $2 \times 10^{3} \mathrm{~T} / \mathrm{m}$ for the trap frequency $0.5 \mathrm{MHz}$.

\section{GENERATION AND DETECTION OF LARGE SPATIAL SUPERPOSITION STATES.}

To prepare spatial quantum superposition state, we need to generate quantum superposition of the nanodiamond at distinct locations. Without the microwave driving, the spin-mechanical coupling Hamiltonian takes the form

$$
H=\hbar \omega_{m} a_{m}^{\dagger} a_{m}+\hbar \lambda S_{z}\left(a_{m}+a_{m}^{\dagger}\right) .
$$

The mechanical mode is initialized to the vacuum state $|0\rangle_{m}$ (or a Fock state $\left|n_{m}\right\rangle_{m}$ ) in a strong trap with the (a) $\quad W$

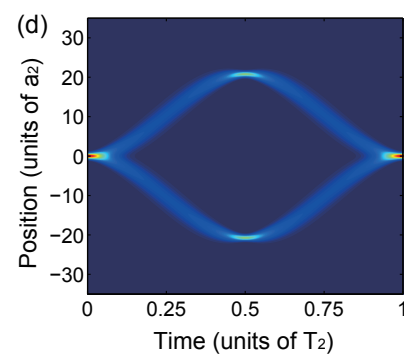

(b)

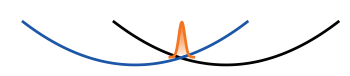

(c)
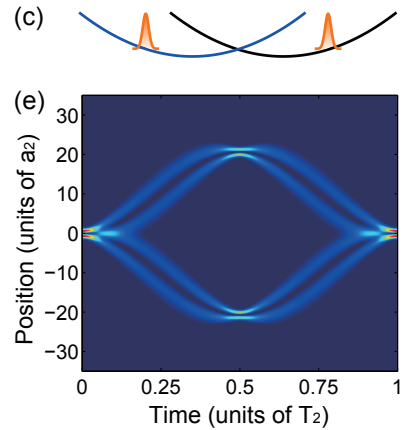

FIG. 3: (Color online) (a) A nanodiamond with $d=30 \mathrm{~nm}$ is confined tightly by a $100 \mathrm{kHz}$ frequency optical tweezer in a magnetic field with a large gradient $G=4 \times 10^{4} \mathrm{~T} / \mathrm{m}$. Its NV center is prepared in a state $|0\rangle$. (b) The power of the optical tweezer is suddenly reduced to $20 \mathrm{kHz}$, and $\mathrm{NV}$ center is changed to a superposition state $(|+1\rangle+|-1\rangle) / \sqrt{2}$, while the magnetic gradient is the same. As a result, the trap centers for different electron spins are separated. (c) The nanodiamond becomes in a spatial superposition state as the state evolves in time. (d) and (e) show the time evolution of the probability distribution of the nanodiamond after the trapping frequency and the NV center state are suddenly changed. The mechanical state is initially $|0\rangle_{m, 100 \mathrm{kHz}}$ (d) or $|1\rangle_{m, 100 \mathrm{kHz}}$ (e) in the high frequency trap. $a_{2}=\sqrt{\hbar / 2 m \omega_{m 2}}=0.092 \mathrm{~nm}$, and $T_{2}=50 \mu \mathrm{s}$.

trapping frequency $\omega_{m 0}$ and the NV center spin is prepared in the state $|0\rangle$. Although the ground state cooling is most effective in a strong trap, to generate large spatial separation of the wave packets it is better to first lower the trap frequency by tuning the laser intensity for the optical trap. While it is possible to lower the trap frequency through an adiabatic sweep to keep the phonon state unchanged, a more effective way is to use a non-adiabatic state-preserving sweep [40], which allows arbitrarily short sweeping time. We denote $\left|n_{m}\right\rangle_{m 1}$ as the mechanical state in the lower frequency $\omega_{m 1}$. We then apply an impulsive microwave pulse to suddenly change the NV spin to the state $(|+1\rangle+|-1\rangle) / \sqrt{2}$ and simultaneously decrease the trap frequency to $\omega_{m 2} \leq \omega_{m 1}$. The evolution of the system state under the Hamiltonian (2) then automatically split the wave packet for the center-of-mass motion of the nanodiamond (see the illustration in Fig. 3). The splitting attains the maximum at time $T_{2} / 2=\pi / \omega_{m 2}$, where the maximum distance of the two wave packets in the superposition state is $D_{m}=8 \lambda a_{2} / \omega_{m 2}=4 g_{s} \mu_{B} G /\left(m \omega_{m 2}^{2}\right)$, where $a_{2}=\sqrt{\hbar / 2 m \omega_{m 2}}$. At this moment, the system state is

$$
\left|\Psi_{S}\right\rangle=\left(|+1\rangle\left|D_{m} / 2\right\rangle_{n_{m}}+|-1\rangle\left|-D_{m} / 2\right\rangle_{n_{m}}\right) / \sqrt{2},
$$

where $\left| \pm D_{m} / 2\right\rangle_{n_{m}} \equiv(-1)^{a_{m}^{\dagger} a_{m}} e^{ \pm D_{m}\left(a_{m}^{\dagger}-a_{m}\right) / 4 a_{2}}\left|n_{m}\right\rangle_{1}$ is the displaced Fock state (or coherent states when 

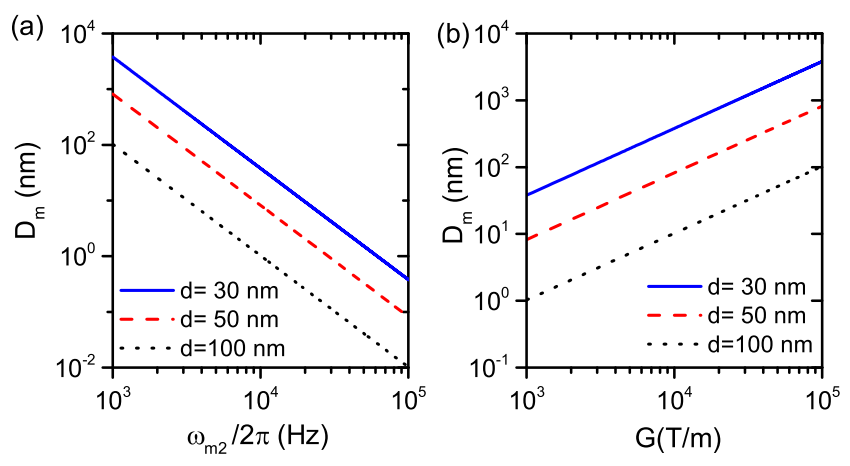

FIG. 4: (Color online) (a) Maximum spatial separation $D_{m}$ of the superposition state as a function of trap frequency $\omega_{m 2}$ when the magnetic gradient is $10^{5} \mathrm{~T} / \mathrm{m}$. (b) Maximum spatial separation $D_{m}$ as a function of the magnetic gradient $G$ when the trapping frequency is $1 \mathrm{kHz}$. Macroscopic superposition states with separation larger than the size of the particle can be achieved with a moderate magnetic gradient.

$\left.n_{m}=0\right)$. This is just the entangled spatial superposition state, as discussed in Appendix A. In Fig. 3 (d) and (e), we show the evolution of the splitting of the spatial wave packets for the nanodiamond under the initial vacuum $|0\rangle_{m}$ or Fock state $|1\rangle_{m}$. The maximum distance $D_{m}$ is plotted in Fig. 4 versus trap frequency, magnetic field gradient, and diameter $d$ of the nanodiamond, and superposition states with separation $D_{m}$ comparable to or larger than the diameter $d$ is achievable under realistic experimental conditions.

To transform the entangled cat state $\left|\Psi_{S}\right\rangle$ to the standard cat state $\left|\psi_{ \pm}\right\rangle_{n_{m}} \equiv\left(\left|D_{m} / 2\right\rangle_{n_{m}} \pm\left|-D_{m} / 2\right\rangle_{n_{m}}\right) / \sqrt{2}$, we need to apply a disentangling operation to conditionally flip the NV spin using displacement of the diamond as the control qubit. This can be achieved as different displacements of the wavepacket induce relative energy shifts of the spin levels due to the applied magnetic field gradient. As an estimate, for the example we considered in Fig. 5 (with a $30 \mathrm{~nm}$-diameter diamond in a 20 $\mathrm{kHz}$ trap under a magnetic gradient of $3 \times 10^{4} \mathrm{~T} / \mathrm{m}$ ), the spin energy splitting is about $2.4 \mathrm{MHz}$ between the $|+1\rangle\left|D_{m} / 2\right\rangle_{n_{m}}$ and $|-1\rangle\left|-D_{m} / 2\right\rangle_{n_{m}}$ components, which is much larger than the typical transition linewidth of the NV spin (in the order of $\mathrm{kHz}$ ). So we can apply first an impulsive microwave pulse to transfer the component state $|+1\rangle\left|D_{m} / 2\right\rangle_{n_{m}}$ to $|0\rangle\left|D_{m} / 2\right\rangle_{n_{m}}$ without affecting $|-1\rangle\left|-D_{m} / 2\right\rangle_{n_{m}}$ and then another pulse to transfer $|-1\rangle\left|-D_{m} / 2\right\rangle_{n_{m}}$ to $\pm|0\rangle\left|-D_{m} / 2\right\rangle_{n_{m}}$. After the two pulses, the spin state gets disentangled and the position of the diamond is prepared in the quantum superposition state $\left|\psi_{ \pm}\right\rangle_{n_{m}}$.

To detect spatial superposition state, we can turn off the optical trap and let the spatial wave function freely evolve for some time $t$. The split wave packets will interference just like the Young's double slit experiment. The period of the interference pattern is $\Delta z=2 \pi \hbar t /\left(m D_{m}\right)$ (Appendix B). As an estimation of typical parameters,
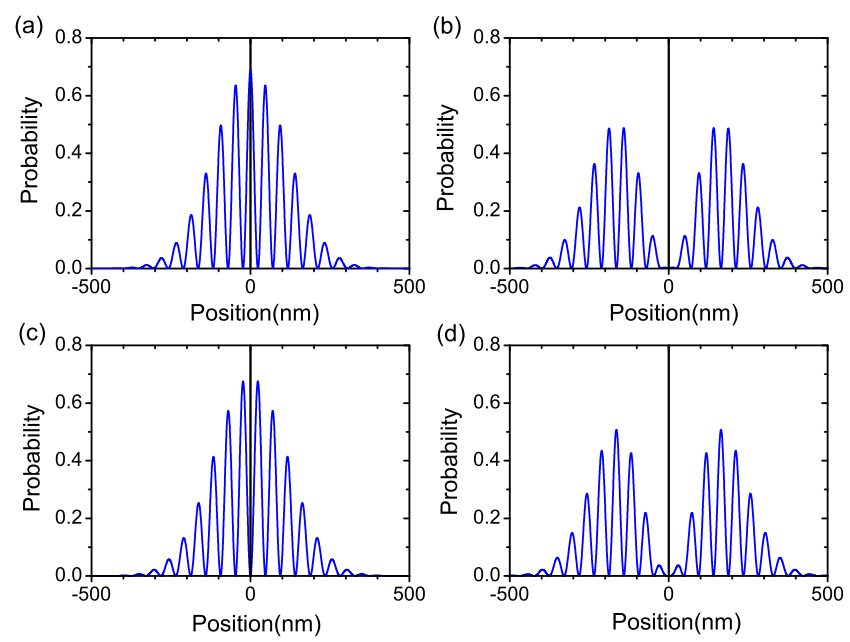

FIG. 5: (Color online) Spatial interference patterns for a $30 \mathrm{~nm}$ nano-diamond after $10 \mathrm{~ms}$ of free expansion. The nanodiamond is initially prepared in the vacuum state $|0\rangle_{m}$ or the 1-phonon state $|1\rangle_{m}$ of a $20 \mathrm{kHz}$ trap. The magnetic gradient is $3 \times 10^{4} \mathrm{~T} / \mathrm{m}$. Before the trap is turned off, the center of mass of the nano-diamond is prepared in (a) $\left|\psi_{+}\right\rangle_{0}$, (b) $\left|\psi_{+}\right\rangle_{1}$, (c) $\left|\psi_{-}\right\rangle_{0}$, and (d) $\left|\psi_{-}\right\rangle_{1}$.

we take $\omega_{m 1}=\omega_{m 2}=2 \pi \times 20 \mathrm{kHz}, d=30 \mathrm{~nm}$, and magnetic field gradient $3 \times 10^{4} \mathrm{~T} / \mathrm{m}$. The spin-phonon coupling rate $\lambda \simeq 2 \pi \times 77 \mathrm{kHz}$ and the maximum distance $D_{m} \simeq 31 a_{2}$. The preparing time of sperposition state is about $25 \mu \mathrm{s}$, which is much less than the coherence time of the NV spin. For the time of flight measurement after turn-off of the trap, we see the interference pattern with a period of $47 \mathrm{~nm}$ after $t=10 \mathrm{~ms}$, as shown in Fig. 5, which is large enough to be spatially resolved [14 16]. In experiments, the initial state is the thermal state with $\left\langle n_{m}\right\rangle \ll 1$, which can be approximated by the vacuum and the first Fock state. By combining the interference pattern of vacuum and $|1\rangle_{m}$ state with its thermal weight, we can get the interference pattern for $n_{m} \ll 1$ thermal state. As show in Fig. [6 because the weight of $|1\rangle_{m}$ is $n_{m} \ll 1$, the final interference patter is very close to the vacuum one. In other words, the interference pattern is robust for the thermal phonon number.

\section{DISCUSSION AND CONCLUSION}

Finally, we briefly mention the source of decoherence for preparation of the spatial quantum superposition state. The decoherence of the cat state by photon scattering is negligible during the time-of-flight measurement as the laser is turned off. The main source of decoherence includes background gas collision and black-body radiation. Using the formula

$$
\gamma_{s}=4 \pi \sqrt{2 \pi} P d^{2} /\left(\sqrt{3} \bar{v} m_{a}\right)
$$

in Ref. 21], we estimate that the decoherence rate due to the background gas collision is about $8 \mathrm{~Hz}$ under the 


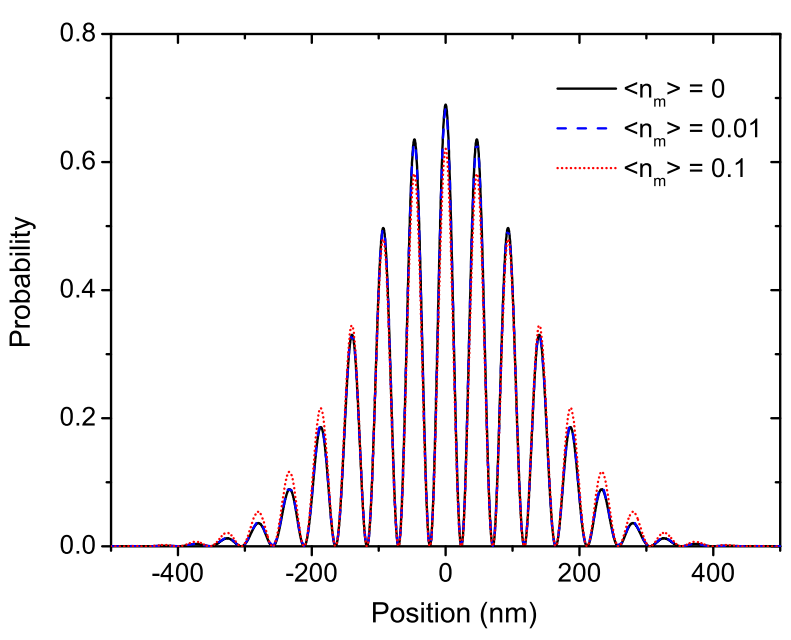

FIG. 6: (Color online) Spatial interference patterns for a $30 \mathrm{~nm}$ nano-diamond after $10 \mathrm{~ms}$ of free expansion. The magnetic gradient is $3 \times 10^{4} \mathrm{~T} / \mathrm{m}$. The nano-diamond is initially prepared in the thermal state of a $20 \mathrm{kHz}$ trap. Before the trap is turned off, the center of mass of the nano-diamond is prepared in the spatial quantum superpostion state with initial thermal phonon number $\left\langle n_{m}\right\rangle=0,0.01,0.1$.

condition that the vacuum pressure $P \sim 10^{-11}$ Torr, the mass of the molecule $m_{a}=4.83 \times 10^{-26} \mathrm{~kg}$, and the gas temperature $T_{b} \sim 4.5 \mathrm{~K}$. Here $\bar{v}=\sqrt{8 k_{B} T /\left(\pi m_{a}\right)}$ is the mean velocity of molcule, $k_{B}$ is Boltzmann constant. This rate is small compared with the experimental time scale of $10 \mathrm{~ms}$. As the internal temperature $T_{i}$ of the diamond is typically much higher than the background gas temperature $T_{b}$, the black-body radiation is dominated by the thermal photon emission from the diamond, which is $\gamma_{b b, e}=\left(2 \pi^{5} / 189\right) c d^{3}\left(k_{B} T_{i} / \hbar c\right)^{6} \operatorname{Im}\left[\left(\epsilon_{b b}-1\right) /\left(\epsilon_{b b}+2\right)\right] z^{2}$ [21]. Here $c$ is the light speed constant, $\epsilon_{b b}$ is the permittivity of diamond for the BB radiation, and $z$ is the interference width. The frequency of the thermal photon is in the order of THz. Compared with the silica sphere considered in 21, the emission rate of $\mathrm{THz}$ photons is smaller by about two orders of magnitude for the diamond. Even with $T_{i}$ at the room temperature, the decoherence rate $\gamma_{b b}$ due to the thermal photon emission is estimated to be only $3 \mathrm{~Hz}$ for our proposed scheme. In addition, with the built-in electronic states of the NV center, the internal temperature of the diamond can be reduced through laser cooling [41]. As $\gamma_{b b} \propto T_{i}^{6}$, this allows further significant suppression of the decoherence rate. For the current discussion of $30 \mathrm{~nm}$ particle in the $0.5 \mathrm{MHz}$ trap, the gravity induced decoherence is estimated to be in the order of $10^{-62} \mathrm{~Hz}$ for the superposition of Fock states [42], and $10^{-7} \mathrm{~Hz}$ for spatial superposition states with displacement $30 \mathrm{~nm}[$ ], and can be neglected. Our proposed scheme is more relevant for testing objective collapse models [21] .

We note that the same scheme could also apply to other optically levitated nano-particles with build-in electron spins, such as ${ }^{28} \mathrm{Si}$ nano-particles with donor spins [43] or nano-crystals doped with rare-earth ions [44]. This scheme thus opens up the possibility to observe large quantum superpositions for mesoscopic objects under realistic experimental conditions and to test predictions of quantum mechanics in unexplored regions.

We thank J.-N. Zhang, K. C. Fong, and S. Kheifets for discussions. This work was funded by the NBRPC (973 Program) 2011CBA00300 (2011CBA00302), NNSFC 11105136, 61033001. LMD acknowledge support from the IARPA MUSIQC program, the ARO and the AFOSR MURI program. TL and XZ were supported by NSF Nanoscale Science and Engineering Center (CMMI0751621).

\section{Appendix A: Derivation of entangled spatial superposition state}

The Hamiltonian (2) in rotating frame with $H_{0}=$ $\hbar \omega_{m} a_{m}^{\dagger} a_{m}$ reads $H_{I}=\hbar S_{z} \lambda\left(a e^{-i \omega_{m} t}+\right.$ h.c. $)$. The unitary operator in the rotating frame is $U_{I}=$ $\mathcal{T} \exp \left[-\frac{i}{\hbar} \int H_{I}(t) \mathrm{d} t\right]$, which can be solved with Magnus expansion $U_{I}=\exp \left[\sum_{k} \Omega_{k}(t)\right]$. Here

$$
\begin{aligned}
\Omega_{1} & =-\frac{i}{\hbar} \int_{0}^{t} H_{I}\left(t_{1}\right) \mathrm{d} t_{1} \\
& =\frac{S_{z} \lambda}{\omega_{m}}\left[\left(e^{-i \omega_{m} t}-1\right) a-\left(e^{i \omega_{m} t}-1\right) a^{\dagger}\right],
\end{aligned}
$$

and

$$
\begin{aligned}
\Omega_{2} & =-\frac{1}{2} \int_{0}^{t} \int_{0}^{t_{1}}\left[H_{I}\left(t_{1}\right), H_{I}\left(t_{2}\right)\right] \mathrm{d} t_{1} \mathrm{~d} t_{2} \\
& =i \lambda^{2}\left(\frac{t}{\omega_{m}}-\frac{\sin \left(\omega_{m} t\right)}{\omega_{m}^{2}}\right) .
\end{aligned}
$$

For $k \geq 3, \Omega_{k}$ are always equal to zero. Neglecting the global phase $\Omega_{2}$, we get $U_{I}=\exp \left[\alpha(t) a-\alpha(t)^{*} a^{\dagger}\right]$, where $\alpha(t)=S_{z} \lambda\left(e^{-i \omega_{m} t}-1\right) / \omega_{m}$. Back to original frame, we get $U_{I}^{\prime}=e^{-i \omega_{m} a_{m}^{\dagger} a_{m} t} U_{I}$. Specifically, at the time of half period with trap frequency $\omega_{m}=\omega_{m_{2}}$, we find that $U_{I}^{\prime}=(-1)^{a_{m}^{\dagger} a_{m}} \exp \left[S_{z} D_{m}\left(a_{m}^{\dagger}-a_{m}\right) / 4 a_{2}\right]$, where $D_{m}=$ $4 g_{s} \mu_{B} G /\left(m \omega_{m_{2}}^{2}\right)$, and $a_{2}=\sqrt{\hbar /\left(2 m \omega_{m_{2}}\right)}$. The entangled spatial state can be calculated as $\left|\Psi_{S}\right\rangle=U_{I}^{\prime}\left|n_{m}\right\rangle_{m 1}$.

\section{Appendix B: Period of interference pattern}

We suppose that the superpostion of displacement state is $\left|\psi_{+}\right\rangle_{0}=\left[\varphi_{0 L}(z)+\varphi_{0 R}(z)\right] / \sqrt{2}$, where $\varphi_{0 R}(z)=$ $\frac{\sqrt{\beta}}{\pi^{1 / 4}} e^{-\beta^{2}(z-b)^{2} / 2}, \varphi_{0 L}(z)=\frac{\sqrt{\beta}}{\pi^{1 / 4}} e^{-\beta^{2}(z+b)^{2} / 2}$, and $\beta=$ $\sqrt{m \omega_{2} / \hbar}=1 /\left(\sqrt{2} a_{2}\right) . b$ is the displacement of the original wavefunction. After the Fourier transfer, the right branch wave function becomes

$$
\varphi_{0 L}(k)=\frac{1}{\pi^{1 / 4} \sqrt{\beta}} e^{-i b k-\left(k^{2} / 2 \beta^{2}\right)} .
$$


The Fourier transfer of the left branch of the wavefunction can be calculated with the similar method. Time evolution of the wavefunction is

$$
\varphi_{0}(z, t)=\frac{1}{\sqrt{2 \pi}} \int_{-\infty}^{+\infty} d k \varphi(k) e^{i(k z-\omega t)}
$$

where $\omega=\hbar k^{2} / 2 m$. We suppose that $z$ in the unit of $1 / \beta, t$ is in the unit of $2 m / \hbar \beta^{2}$, and $k$ is in the unit of $\beta$. The probability distribution of the wave function is

$$
\begin{aligned}
\varphi_{0}^{*}(z, t) \varphi_{0}(z, t) & =\frac{1}{2 \sqrt{\pi\left(1+4 t^{2}\right)}}\left(e^{-\frac{(z-b)^{2}}{1+4 t^{2}}}+e^{-\frac{(z+b)^{2}}{1+4 t^{2}}}\right. \\
& \left.+2 e^{-\frac{z^{2}+b^{2}}{1+4 t^{2}}} \cos \left(\frac{4 b z t}{1+4 t^{2}}\right)\right) .
\end{aligned}
$$

The interference period is $\Delta z=2 \pi\left(1+4 t^{2}\right) /(4 b t)$. For long time limit $b t \gg 1$, we have $\Delta z=2 \pi t / b$. In our paper, we have $D_{m}=2 b$. Therefore, in the S.I. unit the interference period is $\Delta z=2 \pi \hbar t /\left(m D_{m}\right)$.
[1] K. Hornberger, S. Gerlich, P. Haslinger, S. Nimmrichter, and M. Arndt, Rev. Mod. Phys. 84, 157 (2012).

[2] A. Bassi, K. Lochan, S. Satin, T. P. Singh, and H. Ulbricht, Rev. Mod. Phys. 85, 471 (2013).

[3] Yanbei Chen, J. Phys. B: At. Mol. Opt. Phys. 46, 104001 (2013).

[4] S. Nimmrichter, and K. Hornberger, Phys. Rev. Lett. 110, 160403 (2013).

[5] R. Penrose, Gen. Relativ. Gravit. 28, 581 (1996).

[6] C. Davisson and L. H. Germer, Nature (London) 119, 558 (1927); I. Estermann and O. Stern, Z. Phys. 61, 95 (1930); W. Schöllkopf and J. P. Toennies, Science 266, 1345 (1994); M. Kasevich and S. Chu, Phys. Rev. Lett. 67, 181 (1991); C. Monroe et al., Science 272, 1131 (1996); M. Arndt et al, Nature (London) 401, 680 (1999); Gerlich, S. et al, Nat. Commun. 2, 263 (2011).

[7] I. Wilson-Rae, N. Nooshi, W. Zwerger, and T. J. Kippenberg, Phys. Rev. Lett. 99, 093901 (2007).

[8] F. Marquardt, J. P. Chen, A. A. Clerk, and S. M. Girvin, Phys. Rev. Lett. 99, 093902 (2007).

[9] A. D. O'Connell et al, Nature(London) 464, 697 (2010); Jasper Chan et al, Nature(London) 478, 89 (2011); J. D. Teufel et al, Nature(London) 475, 359 (2011).

[10] M. Aspelmeyer, T. J. Kippenberg, F. Marquardt, arXiv:1303.0733 (2013);

[11] D. E. Chang et al., Proc. Natl. Acad. Sci. USA 107, 1005 (2010).

[12] O. Romero-Isart, M. Juan, R. Quidant, and J. Cirac, New J. Phys. 12, 033015 (2010).

[13] O. Romero-Isart et al., Phys. Rev. A 83, 013803 (2011).

[14] T. Li, S. Kheifets, D. Medellin, and M. G. Raizen, Science 328, 1673 (2010).

[15] Tongcang Li, Simon Kheifets, Mark G. Raizen, Nature Phys. 7, 527 (2011).

[16] J. Gieseler, B. Deutsch, R. Quidant, L. Novotny, Phys. Rev. Lett. 109, 103603 (2012).

[17] T. S. Monteiro et al., New J. Phys. 15, 015001 (2013).

[18] A. C. Pflanzer, O. Romero-Isart, and J. I. Cirac, Phys. Rev. A 86, 013802 (2012)

[19] H. K. Cheung, and C. K. Law, Phys. Rev. A 86, 033807 (2012)
[20] Nikolai Kiesel et al., arXiv:1304.6679

[21] O. Romero-Isart et al., Phys. Rev. Lett. 107, 020405 (2011); O. Romero-Isart, Phys. Rev. A 84, 052121 (2011).

[22] Zhang-qi Yin, Phys. Rev. A 80, 033821 (2009).

[23] A. A. Geraci, S. B. Papp, and J. Kitching, Phys. Rev. Lett. 105, 101101 (2010).

[24] A. Arvanitaki, A. A. Geraci, Phys. Rev. Lett. 110, 071105 (2013).

[25] Zhang-qi Yin, Tongcang Li, M. Feng, Phys. Rev. A 83, 013816 (2011).

[26] W. Lechner, S. J. M. Habraken, N. Kiesel, M. Aspelmeyer, P. Zoller, Phys. Rev. Lett. 110, 143604 (2013).

[27] S.J.M. Habraken, W. Lechner, P. Zoller, Phys. Rev. A 87, 053808 (2013).

[28] J. D. Thompson et al., Nature 452, 72 (2008).

[29] J. Wrachtrup and F. Jelezko. Journal of PhysicsCondensed Matter 18, S807 (2006).

[30] V. R. Horowitz et al.. Proc. Natl. Acad. Sci. USA, 109, 13493 (2012)

[31] M. Geiselmann et al.. Nature Nanotechnology 8, 175 (2013)

[32] Levi P. Neukirch et al., Optics Letters 38, 2976 (2013).

[33] C. Tsang et al., IEEE Trans. Magn. 42, 145 (2006).

[34] H. J. Mamin and et al, Nat. Nanotechnol. 2, 301 (2007).

[35] P. Rabl et al., Phys. Rev. B 79, 041302(R) (2009).

[36] S. Kolkowitz et al., Sciene 335, 1603 (2012).

[37] L. Robledo, L. Childress, H. Bernien, B. Hensen, P. F. A. Alkemade, R. Hanson. Nature 477, 574 (2011).

[38] D. M. Meekhof, C. Monroe, B.E. King, W.M. Itano, D.J. Wineland, Phys. Rev. Lett. 76, 1796 (1996).

[39] G. Balasubramanian and et al., Nat. Mater. 8, 383 (2009).

[40] Xi Chen et al, Phys. Rev. Lett. 104, 063002 (2010).

[41] Denis V. Seletskiy et al., Nature Photon. 4, 161 (2010); Jun Zhang et al Nature(Lonon) 493, 504 (2013)

[42] M. P. Blencowe, Phys. Rev. Lett. 111, 021302 (2013).

[43] M. Steger et al., Science 336, 1280 (2012).

[44] W. Tittel et al., Laser \& Photon. Rev. 4, 244, (2010). 